\title{
Adoption of Leanness in the Manufacturing Industry
}

\author{
Jared Landry ${ }^{1}$, Syed Adeel Ahmed ${ }^{2, *}$ \\ ${ }^{1}$ College of Engineering, University of New Orleans, USA \\ ${ }^{2}$ Xavier University of Louisiana, New Orleans, USA
}

Copyright $\mathrm{O} 2016$ by authors, all rights reserved. Authors agree that this article remains permanently open access under the terms of the Creative Commons Attribution License 4.0 International License

\begin{abstract}
Lean manufacturing is a technique that incorporates the elimination of waste, non-value-added activities, and developing a lean workforce. These practices are not implemented into an organization without a committed workforce, upper management leading by example, and the right techniques and management to ensure a smooth transition. Intralox has implemented lean manufacturing and operations into its culture to produce high quality parts efficiently and effectively. They have executed this practice through a lean workforce, leveling inventory, and building in quality to each step of the process. These practices have ensured Intralox is a competitive organization in the plastic manufacturing industry.
\end{abstract}

Keywords Lean Manufacturing, Intralox, Continuous Improvement Cycle, JIT

\section{Introduction}

The manufacturing industry is very diverse and large industry. These industries range from manufacturing components or parts from stock material to molding or casting parts for large assemblies. The molding/casting industry utilized many components and materials to manufacture a separate part that is then sold to the public. Having a strong and lean process that ensures the utilization of base materials and equipment needed to produce parts reduces cost, increases customer satisfaction, and increases efficiency. The material consumption and waste of material is an issue that has constant attention. Utilizing the materials being used and eliminating the majority of waste ensures that the processes being used are considered "lean."

According to the United States Environmental Protection Agency (EPA), "Lean manufacturing is a business model and collection of tactical methods that emphasize eliminating non-value added activities while delivering quality products on time at least cost with greater efficiency."[11] Eliminating non-value added activities increases the efficiency and makes the process stronger in a very competitive market.

Leanness in the manufacturing process is a concept that is being adopted by many companies in recent times. This concept ensures that the processes used, materials consumed and the time spent in manufacturing is most efficient. Ensuring that there is little waste of material and a lean process allows for greater competitiveness in an ever changing market, promotes continuous improvement of all involved and employee empowerment. These concepts not only boost the effectiveness of the company employing lean manufacturing but also the individuals involved.

\section{Literature Review}

There are many principles of lean manufacturing but the main principle would be the reduction or elimination of waste. Waste has many different aspects in manufacturing including the following: over production, unnecessary motion, waste of inventory, defects in production, time spent waiting, transportation time, and over processing. These aspects of waste have little to no added value to the corporation. To combat these non-values adding principles there are techniques and methods that have been proven to eliminate the waste in the manufacturing process.

Continuous improvement plays a critical part in corporations implementing lean manufacturing in their everyday workflow. Carl Wright (2008) wrote, "The Japanese word "kaizen," meaning incremental improvement was formalized by the Toyota Production System, which is now utilized throughout the United States as lean manufacturing."[6] This incremental/continuous improvement practice is vital to companies to streamline work and reduce waste.

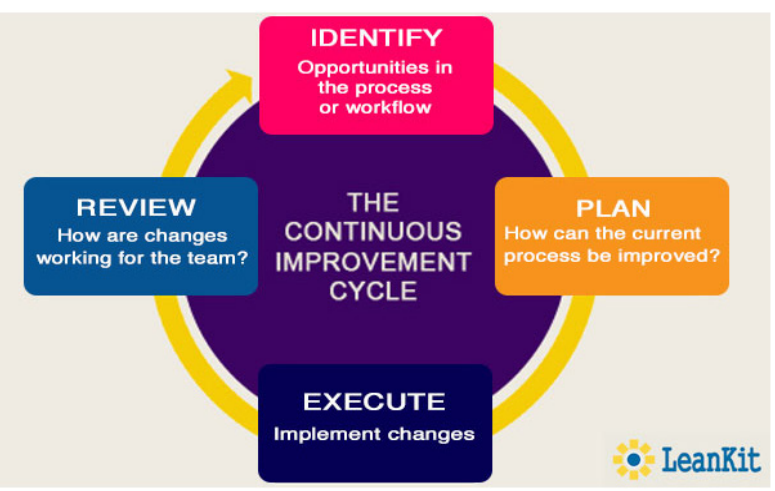

Figure 1. Continuous Improvement Cycle (LeanKit, 2015) 
In the above figure from LeanKit (2015), the continuous improvement cycle can be summarized into four main steps; identify, plan, execute, and review. Identifying opportunities or non-value added steps is vital to the corporation. After identifying these problems, formulating a plan of action to implement these changes is needed. Such plans include just in time approaches, leveled production, and quality built in. Following the plan of action would be to execute the changes that need to be made. Execution is a vital part of the continuous improvement cycle because it involves the entire workforce and managers to be on the same page and trust in the plan to move forward in reducing the waste. Lastly, a review on how the changes are working for the team and to assess how the workflow was affected.

\section{Methods}

Cheng and Podolsky (1993) summarize just-in-time (JIT) manufacturing as, "a Japanese management philosophy applied in manufacturing which involves having the right items of the right quality and quantity in the right place and at the right time." [2] Implementing JIT in manufacturing involves reducing inventory levels to reduce the cost of storing resources that are not used, ensuring that the items used in the manufacturing process are of good quality, and understanding the process time at which products can be produces. Also just in time approaches to manufacturing are only implemented successfully if all employees are engaged and committed to the process. Just in time manufacturing can reduce waste and improve product quality while also making production more efficient and effective if implemented correctly.

Cheng and Podolsky (1993) explain short and long term goals for an organization in implementing JIT manufacturing.[2] These goals include identifying and responding to customer needs, aiming for the optimal quality/cost relationship, eliminating unnecessary wastes, aiming for the development of trusting relationships between the suppliers, designing the plant for maximum efficiency and ease of manufacturing and aiming for continuous improvement even though high standards are already being achieved. Although these goals are set to help implement JIT manufacturing for many companies there are some limitation that may make implementation very difficult for some companies. The use of large inventories to protect against inaccurate forecasts or problems in production is not an aspect of JIT manufacturing and could be difficult for companies to change and still be successful. Also the loss of individual autonomy because of faster cycles and limited slack time in JIT manufacturing could hinder the full acceptance of this approach from all employees.

Another approach to lean manufacturing is leveled production or Heijunka which refers to the production of the same amount of products each day based on forecasts and past ordering history. A key component of this approach is the setting of volume levels for production. This involves creating and studying good forecasts and keeping a good record of order history. Also having sales teams and business unit leaders who are in touch with market place to project and predict sales benefit the company when setting volume levels.

According to Lean-Manufacturing Junction (2008), there are many benefits to implementing leveled production which include the reduction of delivery time, unnecessary overtime, and inventory levels. Also the raw material consumption of the company should be reduced since you are only producing what is need based on the forecast and levels set. Executing Heijunka correctly also reduces the stress level of the workforce because production levels are set and works know in advance what is expected of them.

Lean manufacturing requires processes and production lines to have quality built into them. According to Jon Miller (2003), "Built in quality is the practice of controlling variables within a process, securing quality at one's own process, not passing on poor quality and checking every piece." According to the Toyota Process System, built in quality is structured around four pillars.

- Quality at the source

- Self-checks

- Successive Checks

- $100 \%$ Inspection

The first pillar is to build in quality at the source which means that the quality of the input in the process will determine the quality of the output. The use of quality material, well trained workforce, reliable machines, and proven methods will produce an effective and high quality part. This pillar involves identifying problem when they occur and find the root cause of issue, understanding the quality requirements of the customer and in turn implementing this on the shop floor.

The second pillar is the employment of self-checks which allows for defects to be detected before it move on to the next process. According to Miller (2003), these checks include manual checks or equipment with sensor built in to alert when a defect is detected. Finding the defect when it occurs allows for the narrowing down of the cause variable and the ability to correct the problem before it is hand off to the next process.

The third pillar is the successive check of the part throughout the process. If a defective part is received from a previous process that process is alerted of the defective part. This eliminates the continuous production of defective parts and helps locate the central problem causing poor quality.

The fourth and final pillar in built in quality is the $100 \%$ inspection. This mean that all parts are checked within the whole process to eliminate defects and ensure quality is built in to the process.

A lean workforce is needed to implement just-in-time manufacturing, leveled production and quality built in approaches. Having a workforce that is committed to the lean process, who are customer focused, good problem solvers 
and invested in continuous improvement ensures that the execution of lean manufacturing will be a success. According to Kronos (2003), "Applying lean principles to the workforce can play a critical role in ensuring that labor is aligned to demand, which in turn can result in lower costs and shorter lead times (p. 2)."[1] Identifying non value-added labor, Measuring and managing variability and motivating the workforce are areas that manufactures should focus on to achieve Lean improvements (Kronos). Non value-added labor refers to activities that are worthless to the customer. This could be moving parts from one holding area to another holding area until the parts are assembled. This step adds no value to the product and also hinders the flow of the product thru manufacturing. The next area of focus is on the measuring and managing of variability which refers to the delays caused by absent workers, unscheduled machine downtime or material defects. These variabilities increase costs and escalate lead times. Finally motivating the workforce ensures that workers and the company are in line with the lean process and are working toward a common goal of continuous improvement of the process, individuals and the organization.

Some ways to test the lean approach for some companies is thru the use of computer aided simulations that demonstrate the benefits of lean manufacturing throughout the system. Detty and Yingling give many benefits of using lean manufacturing simulation software which include:

- Warehousing and in-process inventory levels;

- Transport and conveyance requirement;

- The effectiveness of production control and scheduling systems;

- Uniformity of demand and delivery requirements from parts supplier; and

- System responsiveness to market demand and customer delivery requirements. (Detty and Yingling, 2000)

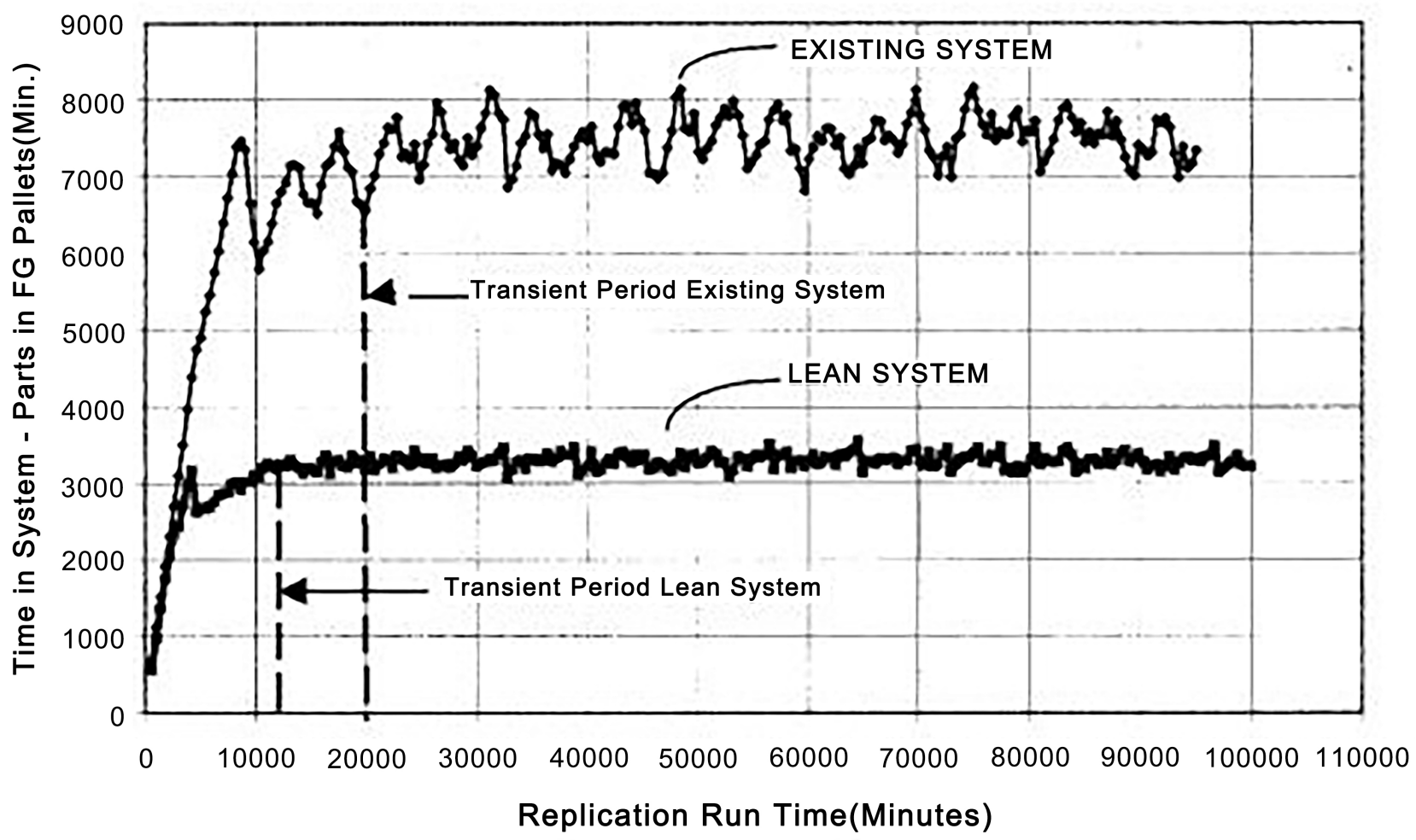

Figure 2. Transient period analysis (Detty and Yingling, 2000)

From the International Journal of Production Research, Detty and Yingling have performed a study on existing and lean systems using computer simulated production facility software. From the figure above you can see that the lean system is about 50\% more efficient than the existing system. This has a lot to do with the start-up or transient period which is twice as long for the existing system as the new lean system.

Based on the series of test that produced the above graph, Detty and Yingling have concluded that the average time parts spend in the system is reduced by $55 \%$ when implementing lean manufacturing techniques. (Detty and Yingling, 2000) The use of lean manufacturing and the increased efficiency of a lean workforce can work together to reduce the inventory levels for a manufacturing facility. Based on a System Modeling Corporation's Arena software package simulation, after comparing existing systems and lean systems it was calculated to have $70 \%$ lower warehouse inventory, $63 \%$ lower exchange inventory, and a 10\% reduction in finished goods inventory. (Detty and Yingling, 2000) This reduction in inventory therefor reduces the amount of floor space required to store parts and work in progress (WIP) by 37\%. (Detty and Yingling, 2000) 


\section{Review}

Intralox employs many of the techniques discussed above to have a lean manufacturing production line. The technique that has the largest impact on lean manufacturing for Intralox is having a lean workforce. Having all of the employees focused on the same goals and shooting for the same outcomes increases productivity, reduces downtime, and increases efficiency. Major steps are taken by managers and employees to generate this lean workforce, including weekly meeting, discussions, and feedback from the employees themselves. Implementing this lean workforce enables Intralox to also adapt other lean manufacturing techniques.

Intralox manages there inventory through leveled quantities as discussed above. These inventory levels are generated from forecasts and sales history that is tracked every year. Inventory levels are then set for each product or product line allowing for some variations in orders. Also this leveled inventory allows manufacturing to run the mold machines more efficiently which reduces the run times of all the machines. With the more efficient running of the machines and more available time to use the machines Intralox offers a four hour guarantee that says that a replacement belt will be shipped within four hours after the order is placed or the belt will be offered at a reduces cost. This guarantee is made available due to lean manufacturing techniques. Employing just in time approach to materials for production, setting leveled inventory, building quality into the part and process, and having a lean workforce allows Intralox to operate very lean and meet all there guarantees.

\section{Conclusions}

The adoption of lean manufacturing in recent years by many companies shows how valuable this process could be to not only manufacturing but to the entire organization. The aspect of lean manufacturing that is most critical for successful implementation would be having focused and integrated workforces who believe that techniques and practices are what is best for the individual and the organization as a whole. Ensuring that workers are heard and able to give feedback of what techniques are beneficial or not allows managers to fine tune the lean practices to better cater to the style and culture of the individual organization. Also having employees who are engaged in this lean technique is the responsibility of the managers. The managers and upper management should practices these techniques to show employees that this is not just another phase the organization is going through but that this is a part of the culture of the organization.

This engaged and lean workforce also allows organizations to easily incorporate another valuable aspect of lean manufacturing known a built in quality. Building in quality into a product starts at the conception of the product or idea and having the correct members of the organization present can eliminate costly downfalls. Receiving input from all aspects of the manufacturing process is valuable to the product being developed and manufactured as lean as possible with very few costly delays or rework. Also investing in process management to ensure the manufacturing process is lean without any non-value added steps allows for more efficient processes and production. Lean manufacturing is great way to increase the value of an organization and increase the competitiveness within its industry.

\section{REFERENCES}

[1] Blogging for Lean disambiguation \& true kaizen | Gemba Panta Rei. (2010, November 28). Retrieved July 20, 2015,

[2] Cheng TCE and Podolsky S, 1993, Just-in-Time Manufacturing - an introduction, Chapman and Hall, London.

[3] Decision Support Tools. (n.d.). Retrieved July 20, 2015,

[4] Detty, R. B., \& Yingling, J. C. (2000). Quantifying benefits of conversion to lean manufacturing with discrete event simulation: a case study. International Journal of Production Research, 38(2), 429-445.

[5] Elnadi, M., \& Shehab, E. (2014, October 31). A Conceptual Model for Evaluating Product-service Systems Leanness in UK Manufacturing Companies. Retrieved July 20, 2015,

[6] Joseph Romm. Lean and Clean Management: How to Boost Profits and Productivity by Reducing Pollution (New York: Kodansha America, 1994), 28

[7] Kaizen: Lean manufacturing continuous improvement. (n.d.). Retrieved July 20, 2015.

[8] Kronos Lean.(n.d.). Retrieved July 20, 2015,

[9] LeanKit. (n.d.). Retrieved July 20, 2015,

[10] Lean Manufacturing and the Toyota Production System. (n.d.). Retrieved July 20, 2015,

[11] Lean Manufacturing Principles. (n.d.). Retrieved July 20, 2015, Levelized Production (Heijunka). (n.d.). Retrieved July 20, 2015 ,

[12] Principles of Lean. (n.d.). Retrieved July 20, 2015, 